\title{
Probing Deformation Mechanisms in Ultrafine Grained Al and Au Thin Films by Quantitative In Situ TEM Deformation
}

\author{
Josh Kacher, Sandra Stangebye, Saurabh Gupta and Olivier Pierron \\ Georgia Institute of Technology, Atlanta, Georgia, United States
}

Understanding dislocation generation mechanisms and interactions with obstacles such as grain boundaries and other dislocations is central to understanding the mechanical behavior of metals and alloys. This has motivated decades of research into the unit processes governing dislocation interactions by in situ transmission electron microscopy (TEM) mechanical testing, resulting in the establishment of basic rules that govern how these interactions occur [1]. However, much of this research has focused on the deformation of coarse-grained polycrystals where the grain boundaries are largely isolated from each other and dislocation glide is primarily transgranular. In ultrafine grained materials, the high volume fraction of grain boundaries can lead to the activation of additional deformation mechanisms, such as grain growth, grain boundary sliding, and intergranular dislocation propagation. This paper presents results on quantitative in situ TEM experiments of deformation of ultrafine grained materials with a focus on deformation-induced grain growth. A unique aspect of this study is the ability to measure the apparent and true activation volumes associated with the material deformation using a micro-electromechanical system (MEMS)-based deformation platform.

Figure 1a-c shows low magnification TEM images of the evolving deformation state of a $200 \mathrm{~nm}$ thick Al sample during tensile deformation. Diffraction analysis of the film showed that the initial sample had a random texture with an average grain size of $90 \mathrm{~nm}$. No dislocation activity was observed, but there was evidence of localized grain growth. Figure 1d-e shows slow grain growth, approximately $0.5 \mathrm{~nm} / \mathrm{s}$. This limited growth was distributed throughout the film and began at early stages of deformation, soon after yield. Grain growth, when occurring, was discontinuous and proceeded in short jumps. Qualitatively, the growth behavior is similar to what has been seen during thermally-induced grain growth in nanocrystalline materials, though with a much lower boundary propagation velocity [2]. At the onset of necking, rapid grain growth, measured to be $6 \mathrm{~nm} / \mathrm{s}$, occurred, leading to the grains more than doubling in size (Fig. 1fg).

Figure 2 shows results from a similar experiment, though conducted on a $100 \mathrm{~nm}$ thick gold film. Transmission Kikuchi diffraction analysis, not shown here for space considerations, showed that the film has a strong $\{111\}$ texture, with an average grain size of $150 \mathrm{~nm}$. In this case, extensive dislocation activity was evident, including both transgranular and intergranular motion. To investigate the effects of intergranular dislocation motion on grain growth, a single grain was tracked during stress relaxation over the course of 5 minutes. In contrast to the deformation behavior of the Al film, grain boundary migration occurred in a continuous fashion over the course of the relaxation experiment, with an average propagation speed of $1 \AA / s$. This grain boundary propagation was accompanied by intergranular dislocation glide and resulted in decreased curvature of the grain boundaries, suggesting that energy minimization may have been a driving force.

The true and apparent activation volumes were measured for both materials by repeated stress relaxation experiments (details of the experiments [3]). This approach has been able to demonstrate a clear difference in activation volume measurements between $\mathrm{Au}$ and $\mathrm{Al}$ (Fig. 2d), suggesting that the approach can be used to differentiate between deformation in different systems. These experiments demonstrate the added utility of combining in situ TEM deformation with quantitative analysis of the deformation behavior, 
facilitating direct comparisons of observed mechanisms and activation volumes across materials systems. This combination of quantitative testing during in situ observation is expected to be especially instrumental in efforts to combine computational and experimental analysis [4]. Future work is focusing on measuring variations of the activation volume with changes in loading conditions, microstructure, and external variables such as ion irradiation.

This work was supported by the U.S. Department of Energy (DOE), Office of Science, Basic Energy Sciences (BES) Materials Science and Engineering (MSE) Division under Award \#DE-SC0018960.

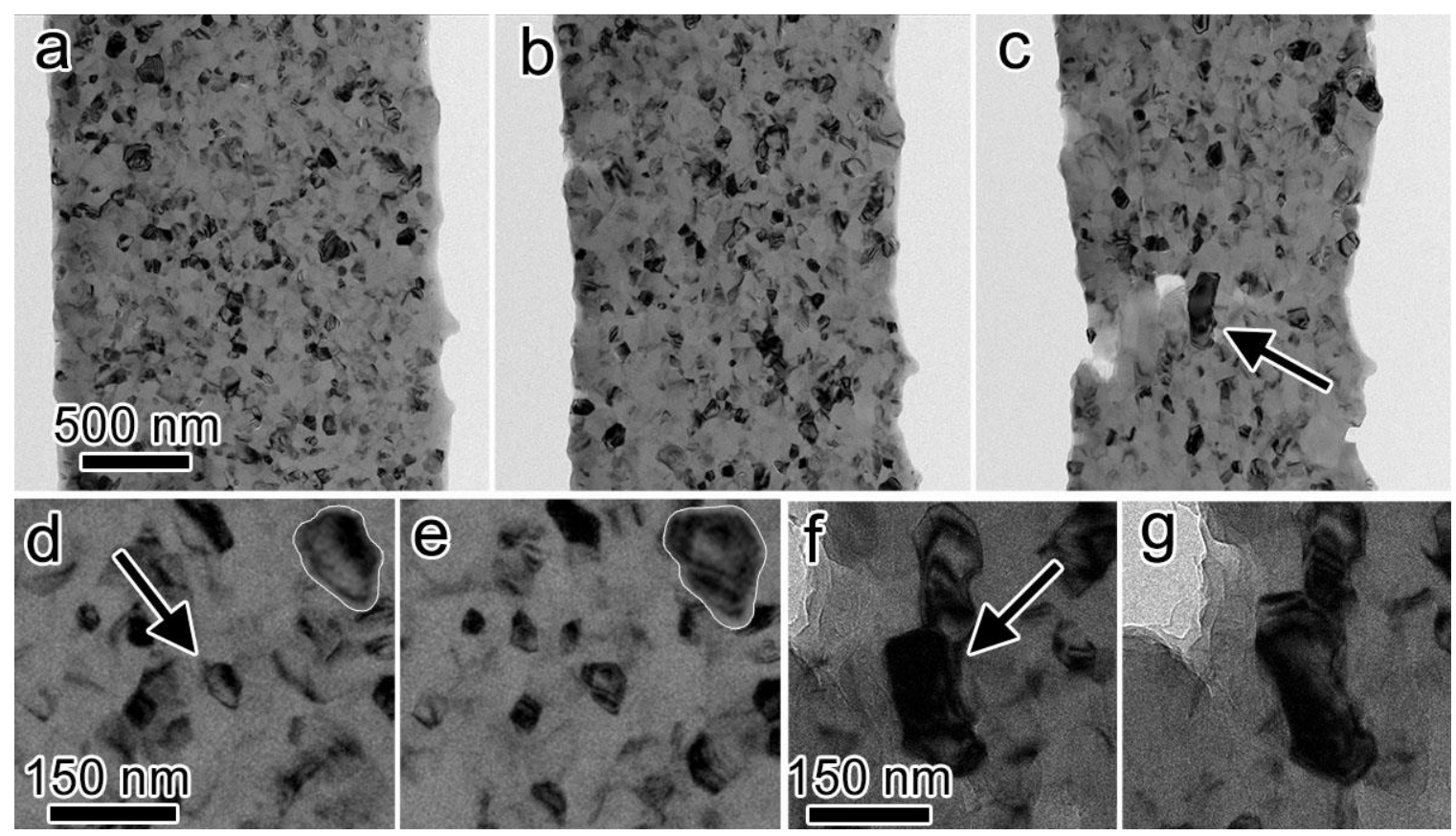

Figure 1. In situ TEM deformation of Al thin film showing bright field TEM images at a) 3\%, b) 10\%, and c) $14.5 \%$ strain. Arrow in (c) indicates enlarged grain in necked region. d-e) Slow grain growth before the onset of necking. Arrow indicates grain of interest. Enlarged images of the grain are shown in the insets. Frames were captured $34 \mathrm{~s}$ apart. f-g) Rapid grain growth in necked region. Arrow indicates grain of interest. Frames were captured approximately $16 \mathrm{~s}$ apart. 

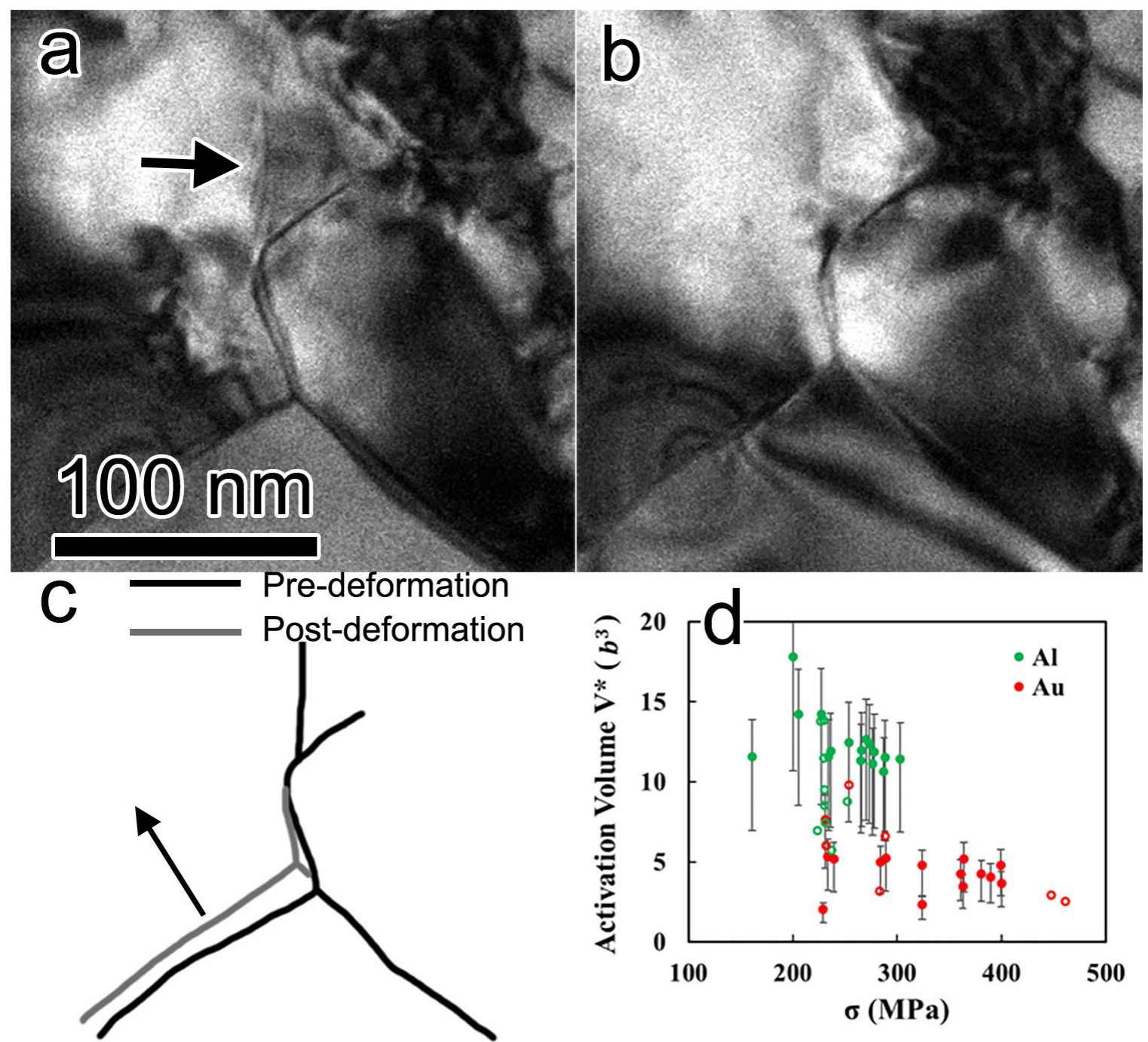

Figure 2. Grain growth during in situ TEM deformation of gold thin film. a-b) BF TEM images captured 5 minutes apart. Arrow indicates dislocations gliding in twin boundary plane. c) Trace of grain boundaries before and after deformation. Arrow indicates grain boundary propagation direction. d) Activation volume measurements as a function of stress for $\mathrm{Al}$ and $\mathrm{Au}$.

References

1. Kacher, J., et al. Current Opinion in Solid State and Materials Science, 2014. 18(4): p. 227-243.

2. Kacher, J., et al. Materials Science and Engineering A, 2011. 528(3): p. 1628-1635.

3. Gupta, S. and O.N. Pierron, Journal of Microelectromechanical Systems, 2017. 26(5): p. 1082-1092.

4. Kacher, J., et al. Current Opinion in Solid State and Materials Science, 2019. 23(3): p. 117-128. 